\title{
Land Information Extraction with Boundary Preservation for High Resolution Satellite Image
}

\author{
Suresh Singh \\ Geomatics Engg. Group \\ IIT, Roorkee \\ Roorkee, India
}

\author{
Merugu Suresh \\ Geomatics Engg. Group \\ IIT, Roorkee \\ Roorkee, India
}

\author{
K. Jain \\ Geomatics Engg. Group \\ IIT, Roorkee \\ Roorkee, India
}

\begin{abstract}
The advancement of technology in satellite system has drastically improved the quality of images which we call high resolution images. Today we have many satellites which provide high resolution images such as QUICKBIRD, IKONOS,WORLD-VIEW etc. High resolution provides much greater detail of information such as buildings or trees etc. can be seen clearly. Now the question arises how we can extract these land objects which contain various information. Traditionally we use manual digitization which is a time taking task and not appropriate for the changing land details.

In this modern world we need some fast techniques which can extract the land boundaries as well as give the information associated with them such as their area. Object based techniques are used for the high resolution images but it is associated with the problem of proper segmentation. This paper includes efficient technique for edge detection to define land boundaries and feature selection technique for land information extraction. So this paper aims to use an edge detection technique and object based classification to extract the land information automatically and then associate the area detail with each land object.
\end{abstract}

\section{Keywords}

Land Parcel, Edge Detection, Object, Feature Selection, Segmentation

\section{INTRODUCTION}

Extracting land information has been a challenging task for the modern world as there is no proper method available for this. We often use manual digitization to extract land objects and use their database information for further work. This approach is time taking and with change in the land detail updating the information is very challenging task. Object based techniques are the solution of this problem which was derived from the necessity of their use in the high resolution images. Earlier we were using pixel based techniques for all images to extract the information but in case of high resolution images pixel based techniques cannot be applied as two different objects may have same reflectance value [1\&2].The problem associated with object based classification is the quality of segmentation which is very necessary to extract the land objects so that proper information can be retrieved. Segmentation result can be improved with the help of edge detection techniques. Today we have different edge detecting methods but for better result we will apply a relatively new method for edge detection which is helpful in better segmentation.
So our first aim will be edge detection which can be used as one of the image layer during segmentation. Then we will use object based techniques to separate these objects.

\section{LITERATURE REVIEW}

There are many methods available for classification of images but in case of high resolution images pixel property is not sufficient to classify the image. Earlier pixel based hard and soft classifications were used but use of the image interpretation elements can create more distance between various land features available on the image. This paper includes an object based technique to classify the image. Here different land features were classified and the overall accuracy was near $82 \%$ which can be increased by using more image interpretation elements [3\&12].

This paper contains various segmentation methods of object based analysis which includes three basic approaches i.e. region approach, boundary approach and edge approach. Segmentation is a procedure of defining the objects. In segmentation the whole image got segmented in the form of objects. Here comparison between these segmentation algorithms was shown which helps in selection of segmentation algorithm [10\&11].

After this there is need of edge detection so that boundary of object can be identified. Traditionally a number of methods are available which include Canny, Sobel Laplace etc. But these methods can be modified with the use of directionality and efficient thresholding algorithms [6].

\section{STUDY AREA AND DATA}

The study area is located in Burghausen, Germany which is a Rapid Eye image with 5m resolution.

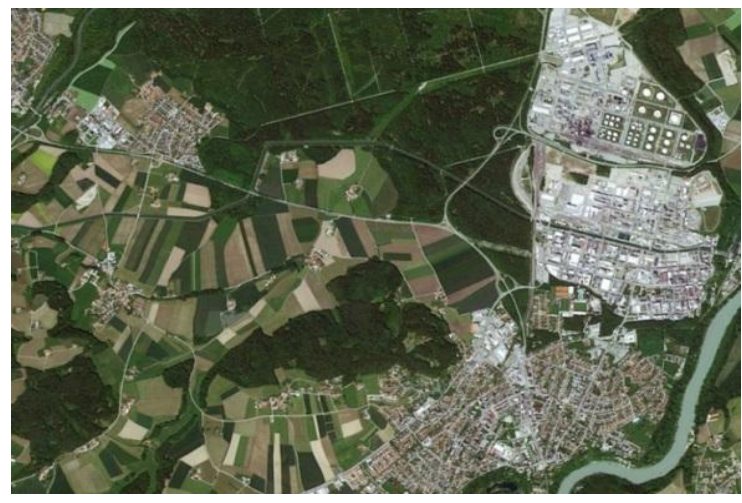

Fig 1: Study Area

Please use a 9-point Times Roman font, or other Roman font with serifs, as close as possible in appearance to Times This 
image contains various details such as buildings, roads, agricultural fields, forest area, river which we have to extract with their proper boundary.

\section{METHODOLOGY}

\subsection{Edge Detection Technique}

This is the most important part of the land information extraction. Edge detection means to detect the pixel changing boundaries [9\&13]. We have different methods available for edge detection but in case of satellite images these techniques are not very promising as land objects do not have very sharp boundary[14]. So for better result we will use this new edge detection algorithm which is written in MATLAB.

In this method we modify the Sobel operator. As we know that Sobel operator only detect horizontal and vertical edges and find the gradient by them but this is not the solution for satellite images where we have effect of noise and texture variation. So we detect gradient at horizontal, vertical, 450 and 1350 by using 5 X 5 mask

$H x=$\begin{tabular}{|l|l|l|l|l|}
\hline & 2 & 3 & 2 & 1 \\
\hline 2 & 3 & 4 & 3 & 2 \\
\hline 0 & 0 & 0 & 0 & 0 \\
\hline-2 & -3 & -4 & -3 & -2 \\
\hline-1 & -2 & -3 & -2 & -1 \\
\hline
\end{tabular}

Hy $=$\begin{tabular}{|c|c|c|c|c|}
\hline 1 & 2 & 0 & -2 & -1 \\
\hline 2 & 3 & 0 & -3 & -2 \\
\hline 3 & 4 & 0 & -4 & -3 \\
\hline 2 & 3 & 0 & -3 & -2 \\
\hline 1 & 2 & 0 & -2 & -1 \\
\hline
\end{tabular}

$\mathrm{H}_{45}=$\begin{tabular}{|l|l|l|l|l|}
\hline $\mathbf{3}$ & $\mathbf{2}$ & $\mathbf{1}$ & $\mathbf{2}$ & 0 \\
\hline $\mathbf{2}$ & $\mathbf{4}$ & $\mathbf{3}$ & 0 & -2 \\
\hline 1 & 3 & 0 & -3 & -1 \\
\hline 2 & 0 & -3 & -4 & -2 \\
\hline 0 & -2 & -2 & -3 & -3 \\
\hline
\end{tabular}

$\mathrm{H}_{135}=$\begin{tabular}{|l|l|l|l|r|}
\hline 0 & -2 & -1 & -2 & -3 \\
\hline 2 & 0 & -3 & -4 & -2 \\
\hline 1 & 3 & 0 & -3 & -1 \\
\hline 2 & 4 & 3 & 0 & -2 \\
\hline 3 & 2 & 1 & 2 & 0 \\
\hline
\end{tabular}

Fig 2: Mask for Edge Detection

So the highest gradient output will be selected and then we use threshold value using Otsu algorithm [10]. We can see the result by this method which will be used in further work.

\subsection{Comparison of Result}

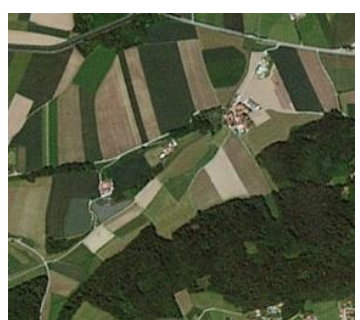

(a)

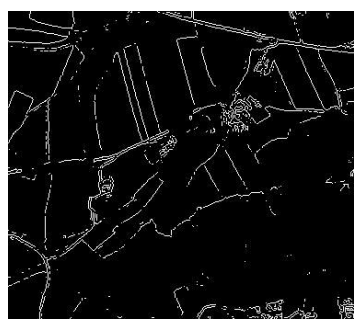

(c)

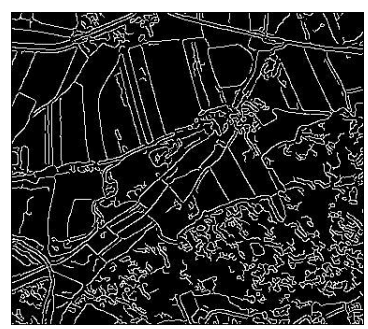

(b)

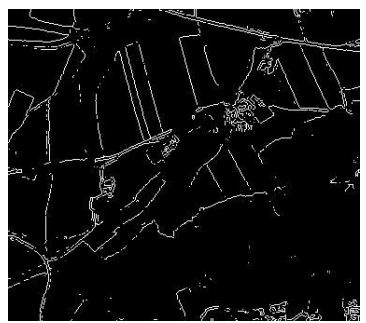

(d)

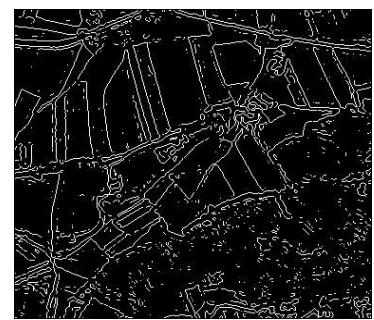

(e)

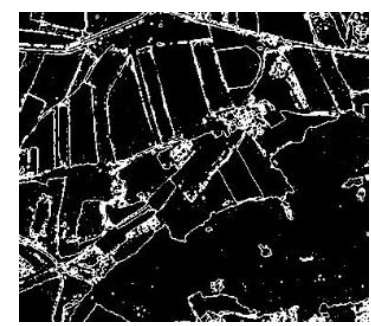

(f)

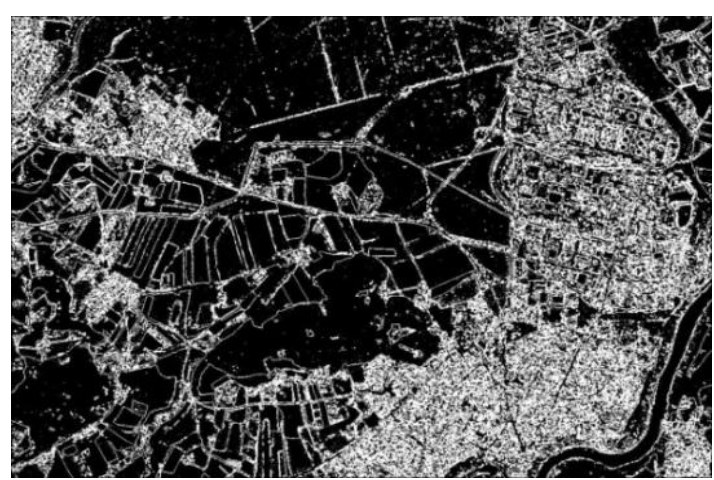

(g)

Fig 3: Edge Detection Results (A). Test Image (B). Canny Edge Detection (C). Prewitt Edge Detection (D). Sobel Edge Detection (E). Laplace Edge Detection (F). Modified Method Edge Detection (G). Overall Edge Detection with Modified Method

\subsection{Object Based Analysis for Land Object Definition}

Here we have used e-Cognition Developer 8.1 to define the land objects. In first step we will merge the edge detection result with the original image and then we will apply the multiresolution segmentation with a scale to define the objects boundary [5].

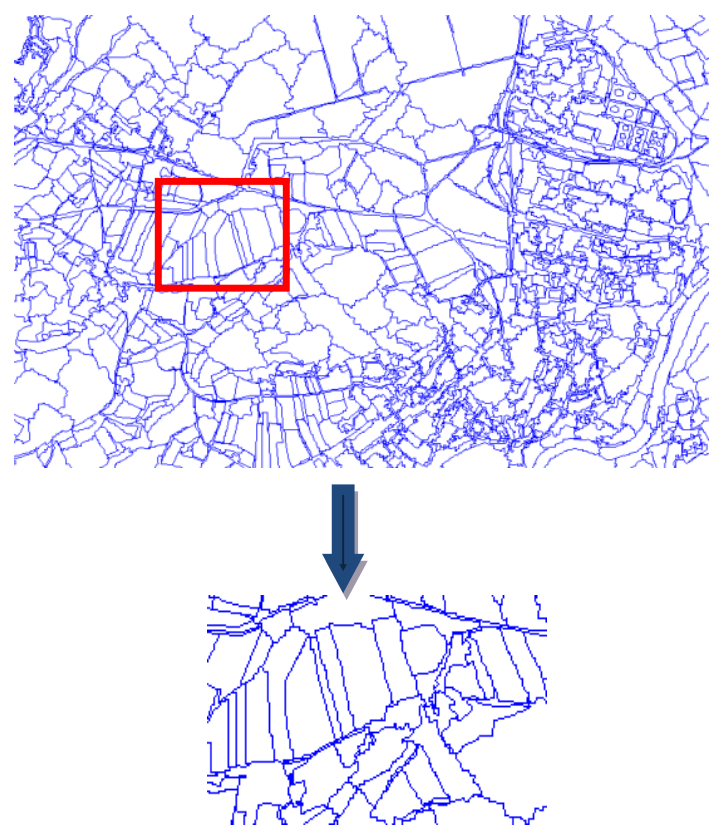

Fig.4 Land object boundary result after Segmentation 


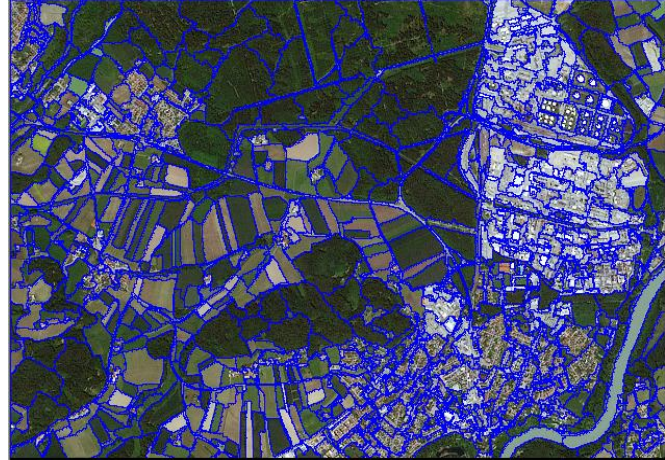

Fig.5 Segmentation Result

\section{LAND INFORMATION} EXTRACTION RESULT

After applying the above steps Rule Based Algorithms are written in e-Cognition which is based on the Feature Selection. Here we have 5 classes i.e. Forest, River, Roads, Agriculture Fields Buildings. The Rule Based Algorithms are written with each land class extraction.

So the land information results are as follows:

\subsection{Forest Extraction}

a. Object with Brightness $<60$, Assign Class $=$ Forest

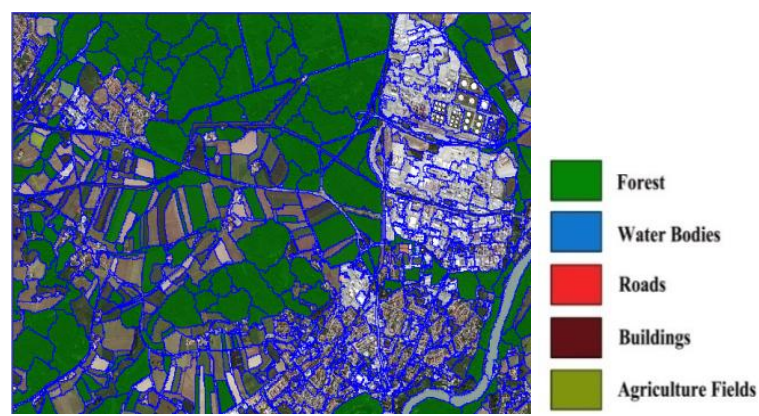

Fig.6 Forest Extraction Result

\subsection{River Extraction}

a. Object with Brightness value- $132<$ Brightness $<138$

Assign Class=River

b. River object with Standard Deviation (S.D)> 30

Marked Unclassified

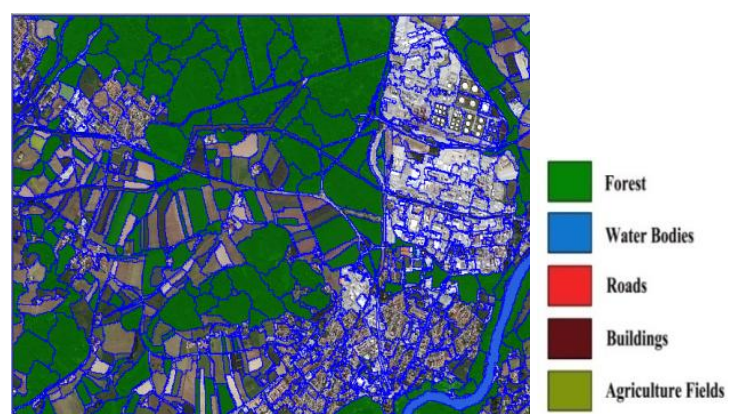

Fig.7 River Extraction result

\subsection{Roads Extraction}

a. $\quad$ Object with Elliptical Fit $<0.1$

Assign Class=Roads b. Merge Road Object and

Road with area < 11000 Pixel Marked as Unclassified

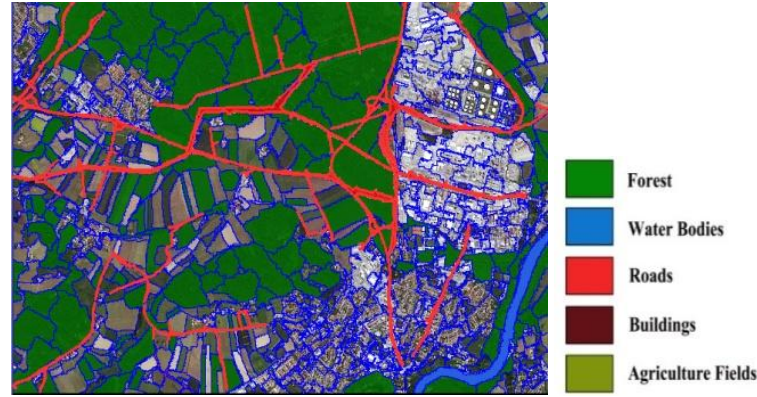

Fig.8 Road Extraction Result

\subsection{Agriculture Fields Extraction}

a. Unclassified Object with Brightness < 175 and S.D. $<30$

Assign Class=Agriculture Fields

b. Forest Object with Asymmetry $>0.89$

Assign Class $=$ Agriculture Fields

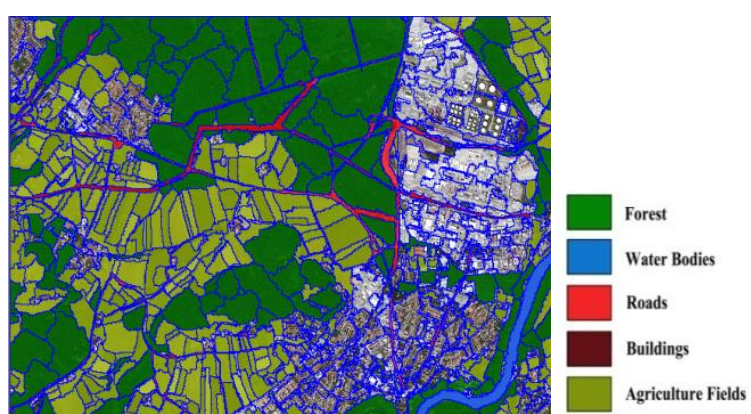

Fig.9 Agriculture Fields Extraction Result

\subsection{Buildings Extraction}

a. Table 1: Feature Selection

Assign Class $=$ Buildings

b. Merge Object assigned as Buildings and Forest

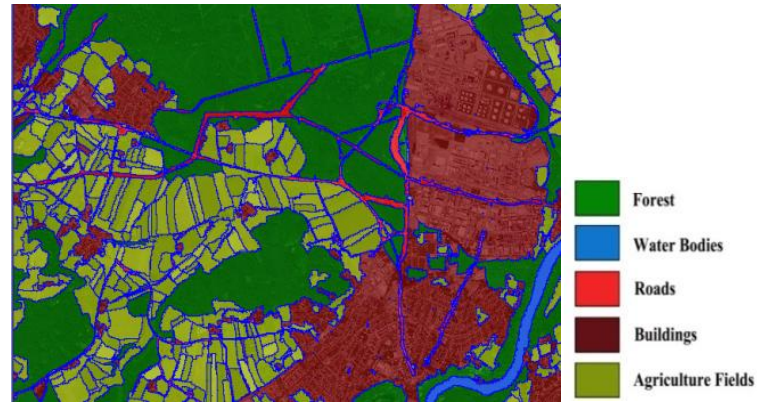

Fig.10 Final Result

So overall following Features can be selected for Land Information Extraction. Here Qualitative Knowledge refers to the user knowledge and Quantitative Knowledge is the mathematical form of Quantitative Knowledge which can be used for Feature Extraction. 
Table 1: Feature Selection

\begin{tabular}{|l|l|l|}
\hline Class & $\begin{array}{l}\text { Qualitative } \\
\text { Knowledge }\end{array}$ & Quantitative Knowledge \\
\hline River & $\begin{array}{l}\text { Color, } \\
\text { Texture }\end{array}$ & Reflectance, Standard Deviation \\
\hline Forest & Color & Reflectance \\
\hline Roads & Shape & Very small Elliptical fit \\
\hline $\begin{array}{l}\text { Agriculture } \\
\text { Fields }\end{array}$ & $\begin{array}{l}\text { Shape, } \\
\text { Texture }\end{array}$ & Reflectance, Standard deviation \\
\hline Buildings & $\begin{array}{l}\text { Color, } \\
\text { Shape }\end{array}$ & $\begin{array}{l}\text { Higher Brightness, } \\
\text { High Rectangular fit, Elliptical } \\
\text { fit }\end{array}$ \\
\hline
\end{tabular}

\section{RESULT AFTER BOUNDARY SMOOTHNESS}

For better visual representation the raster boundary can be converted into vector with the help of direct algorithm available in e-Cognition Developer.
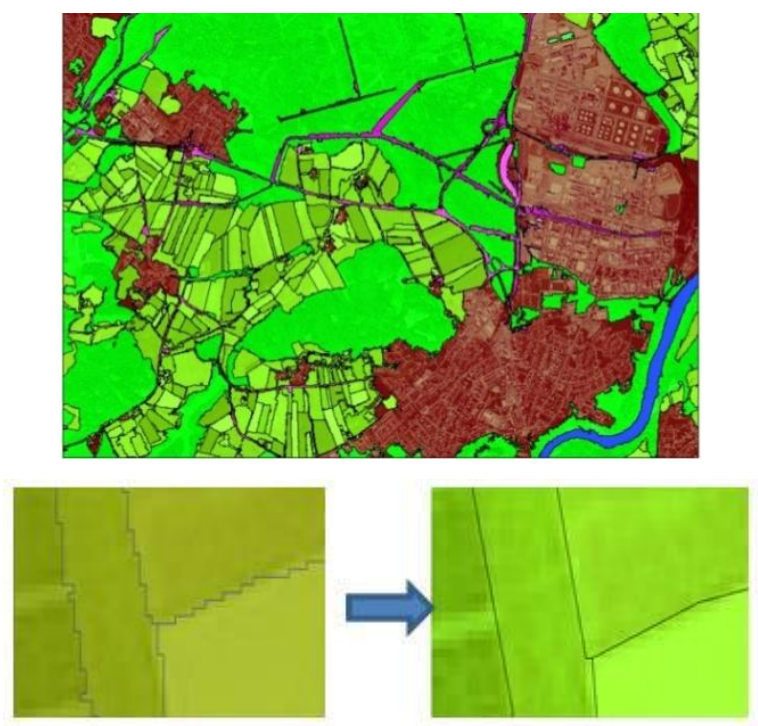

Fig.11 Result after Boundary Smoothness

\section{ERROR MATRIX}

The accuracy of this method can be checked with the help of Accuracy Assessment matrix which is given as follows:

Table 2: Error matrix

\begin{tabular}{|l|l|l|l|l|l|l|}
\hline & Buildings & $\begin{array}{l}\text { Agriculture } \\
\text { fields }\end{array}$ & Forest & Road & River & Total \\
\hline Buildings & $\mathbf{1 0 3}$ & 5 & 4 & 2 & 0 & $\mathbf{1 1 4}$ \\
\hline $\begin{array}{l}\text { Agricultu } \\
\text { re fields }\end{array}$ & 2 & $\mathbf{1 3 9}$ & 2 & 2 & 0 & $\mathbf{1 4 5}$ \\
\hline Forest & 4 & 1 & $\mathbf{1 5 3}$ & 0 & 0 & $\mathbf{1 5 8}$ \\
\hline Road & 3 & 1 & 4 & $\mathbf{4 3}$ & 0 & $\mathbf{5 1}$ \\
\hline River & 0 & 0 & 0 & 0 & $\mathbf{3 2}$ & $\mathbf{3 2}$ \\
\hline Total & $\mathbf{1 1 2}$ & $\mathbf{1 4 6}$ & $\mathbf{1 6 3}$ & $\mathbf{4 7}$ & $\mathbf{3 2}$ & $\mathbf{5 0 0}$ \\
\hline
\end{tabular}

Overall Classification Accuracy $=94 \%$

Kappa coefficient $=0.919$

\section{APPLICATION (AREA INFORMATION DETAIL)}

One of the most important applications of this land information extraction is that we can associate each land object with their area which formed automatically during segmentation. The area shown here is in meters.
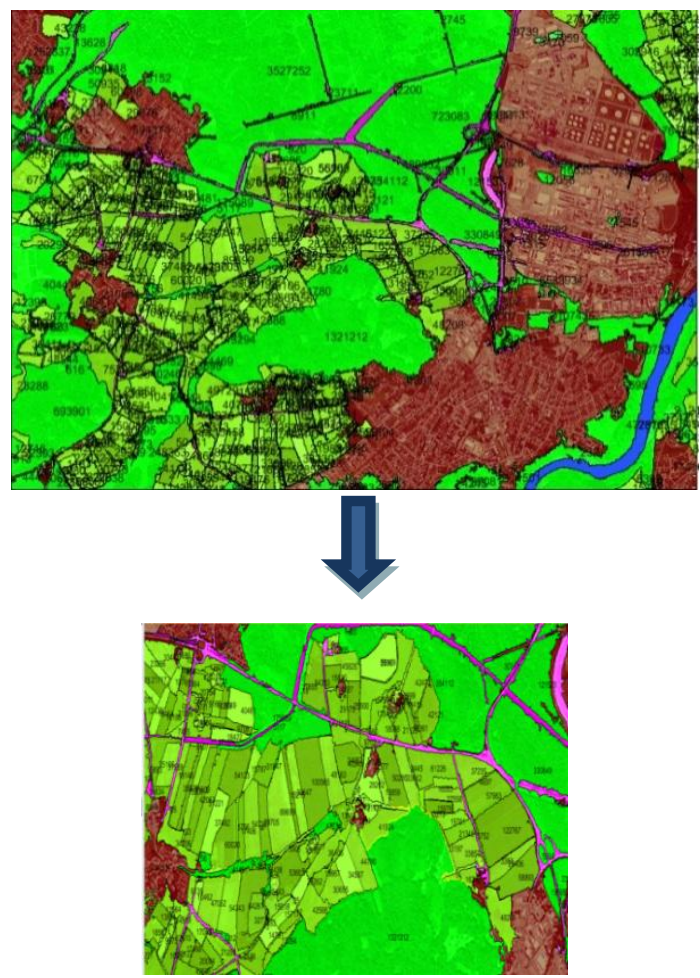

Fig.12 Area Information Result

\section{CONCLUSION}

From this analysis we can observe that information from high resolution image can be extracted which solves the problem of feature extraction. Pixel based techniques are only confined to resource mapping and not suitable for information extraction from high resolution images which are basic input for detailed mapping. The proposed method gives the alternative for information extraction from such images with preserving land object boundary by utilizing edge detection in which one can observe that traditional methods for edge detection didn't shows much better results for satellite images as satellite images have complex texture and it doesn't contain sharp boundaries. The use of directionality and window size in edge detection improves the boundary extraction result and thus directional Sobel $5 \times 5$ edge detection result is taken which works as one of the image layer during segmentation. This modification is the basic input for object based analysis because without appropriate segmentation we will not be able to extract the land objects. So this segmentation technique can make the segmentation result satisfactory which leads to better results. The use of feature selection by merging the qualitative and quantitative knowledge defines the rule sets thus the object based analysis shows that automatic land information extraction is possible with preservation of boundary. One can simply use the distinguishable properties to write the algorithm for each land class. 
The future scope of the project is to minimize the computation time for segmentation process with the help of efficient parallel processing algorithms for fast execution and efficient memory utilization. From this method a database can be created for land records which can help different government or private organization to access the information.

So overall we can say that this technique can be used as an alternative of manual digitization which can use for detailed mapping for high resolution images while preserving the boundary of land objects and helps in fast information retrieval.

\section{REFERENCES}

[1] Anuj Tiwari, Merugu Suresh, Arun Kumar Rai, (2014), Ecological Planning for Sustainable Development with a Green Technology: GIS, International Journal of Advanced Research in Computer Engineering \& Technology (IJARCET), Volume 3 Issue 3, March 2014, ISSN: 2278 - 1323, pp 636-641.

[2] Arora S. Acharya J, Verma A, Panigrahi Prasanta K. (2007), Multilevel thresholding for image segmentation through a fast statistical recursive algorithm, Indian Institute of Technology, Kharagpur 721 302, Indiac Physical Research Laboratory, Navrangpura, Ahmedabad 380 009, India.

[3] Belgiu Mariana, Lampoltshammer Thomas J. (2013), Ontology based interpretation of very high resolution imageries grounding ontology on visual interpretation keys, Salzburg Department of Geoinformatics Schillerstrasse 30, 5020 Salzburg, Austria.

[4] Bouziani Mourad, Goita Kalifa (2010), Rule-Based Classification of a High Resolution Image in an Urban Environment Using Multispectral Segmentation Guided by Cartographic Data, IEEE transactions on geoscience and remote sensing, vol. 48 , no. 8 .

[5] Cleve Casey, Kelly Maggi, Kearns Faith R., Moritz Max (2007), Classification of the wildland-urban interface: A comparison of pixel- and object-based classifications using high-resolution aerial photography, Center for Fire Research and Outreach, University of California, Berkeley, 137 Mulford Hall \#3114, Berkeley, CA 94720, United States.

[6] Caetano Mario (2009), Image classification, Department of Geology,University of Prague,Czech Republic.

[7] G.T. Shrivakshan (September 2012), A Comparison of various Edge Detection Techniques used in Image Processing, Bharathiar University, Coimbatore, Tamilnadu, India, IJCSI International Journal of Computer Science Issues, Vol. 9, Issue 5, No 1.
[8] Jabari Shabnam and Zhang Yun (2013), Very High Resolution Satellite Image Classification Using Fuzzy Rule-Based Systems, Department of Geodesy and Geomatics Engineering, University of New Brunswick, 15 Dineen Drive, Fredericton, NB E3B 5A3, Canada.

[9] Karlsson Anders (2003),Classifcation of high resolution satellite images, Chalmers University of Technology SE41296 GAoteborg, Sweden.

[10] Lu D. and Weng Q. (2006), A survey of image classification methods and techniques for improving classification performance, Department of Geography, Geology, and Anthropology, Indiana State University, Terre Haute, IN 47809, USA.

[11] Merugu Suresh, Kamal Jain, (2014), A Review of Some Information Extraction Methods, Techniques and their Limitations for Hyperspectral Dataset International Journal of Advanced Research in Computer Engineering \& Technology (IJARCET), Volume 3 Issue 3, March 2014, ISSN: 2278 - 1323, pp 2394-2400.

[12] Merugu Suresh, Kamal Jain, (2013) Sub Pixel Analysis on Hypothetical Image by using Colorimetry, International Journal of Recent Technology and Engineering(IJRTE), ISSN: 2277-3878,Volume 2, Issue4, September 2013.

[13] Merugu Suresh, Kamal Jain, (2013), Colorimetrically Resolution Enhancement Method for Satellite Imagery to Improve Land Use, 14th ESRI User Conference id: UCP0046, New Delhi, India, 11-12th Dec, 2013.

[14] Merugu Suresh, Kamal Jain, (2014), A New Super Resolution Mapping Algorithm by Combining Pixel and Subpixel-Level Spatial Dependences with Colorimetry, Journal of Indian Society of Remote Sensing (ISRS), 10/2014; 42(4):10, Springer.

[15] Narkhede H.P. (2013), Review of Image Segmentation Techniques, International Journal of Science and Modern Engineering (IJISME) ISSN: 2319-6386, Volume-1, Issue-8.

[16] Syed Sohel, Dare Paul, Jones Simon (2003), Automatic classification of land cover features with high resolution imagery and lidar data: an object-oriented approach, The university of California,USA.

[17] Vala Hetal J., Baxi Astha (February 2013), A Review on Otsu Image Segmentation Algorithm, Department of Computer Engineering Parul Institute of Engineering \& Technology, Waghodia, Gujarat (India). International Journal of Advanced Research in Computer Engineering \& Technology (IJARCET) Volume 2, Issue 2. 